\title{
Baseline predictors of progression of Parkinson's disease in a sample of Egyptian patients: clinical and biochemical
}

\author{
Asmaa Helmy', Eman Hamid', Mohamed Salama ${ }^{2,3}$, Ahmed Gaber ${ }^{1}$, Mahmoud El-Belkimy ${ }^{1}$ and Ali Shalash ${ }^{{ }^{*}}$ (B)
}

\begin{abstract}
Background: Clinical progression of Parkinson's disease (PD) is highly heterogeneous, and its predictors are generally lacking. Identifying predictors of early disease progression is important for patients' management and follow-up. The current study aims to identify clinical, neuroimaging and biochemical baseline predictors of motor progression in patients with PD. Forty-five PD patients were assessed at baseline, 6 months and 1 year using MDS-UPDRS total and subscores, Hoehn and Yahr (H\&Y), Schwab and England (S\&E), International Physical Activity Questionnaire (IPAQ). Baseline New Freezing of Gait Questionnaire (NFOG-Q), Berg Balance Scale (BBS), Ten-Meter Walking Test (10-MWT), and Time Up and Go Test (TUG), Non-Motor Symptoms Scale (NMSS), Beck Depression Inventory (BDI), PD questionnaire 39 (PDQ-39), MRI brain, uric acid, lipid profile and glycated hemoglobin were performed.

Results: Significant worsening of MDS-UPDRS total, part III scores, H\&Y, S\&E and IPAQ $(p<0.001)$ was detected. One-year progression of H\&Y and S\&E were significantly correlated to disease duration $(p=0.014, p=0.025$, respectively). Progression of H\&Y was correlated to baseline TUG $(p=0.035)$. S\&E progression was correlated to baseline MDS-UPDRS total score (rho $=0.478, p=0.001)$ and part III (rho $=0.350, p=0.020), \mathrm{H} \& \mathrm{Y}(\mathrm{rho}=0.401, p=0.007), \mathrm{PIGD}$ $(\mathrm{rho}=0.591, p<0.001)$, NFOG-Q $(\mathrm{rho}=0.498, p=0.001)$, and TUG $(\mathrm{rho}=0.565, p=0.001)$. Using linear regression, there was no predictors of clinical progression among the used baseline variables.
\end{abstract}

Conclusion: Despite the significant motor and physical activity progression over 1 year that was correlated to baseline motor and gait severity, but without predictive value, further similar and longitudinal studies are warranted to detect predictors of early progression and confirm findings.

Keywords: Parkinson's disease, Predictors, Progression, Egyptian, COVID-19

\section{Background}

Parkinson's disease (PD) is the second most common progressive neurodegenerative disorder, affecting $1 \%$ of people older than 60 years, with incidence rates of $8-18$ per 100,000 person-years in prospective populationbased studies [1]. PD is a complex disease with clinical, genetic, and molecular grounds. Clinical progression is therefore highly heterogeneous across individuals, and

\footnotetext{
*Correspondence: ali_neuro@yahoo.com; drali_shalash@med.asu.edu.eg ${ }^{1}$ Department of Neurology, Faculty of Medicine, Ain Shams University, 168 Elnozha St, Saint Fatima Square, Heliopolis, Cairo, Egypt Full list of author information is available at the end of the article
}

predictors of individual progression are generally lacking $[2,3]$.

Most studies on the annual rate of changes of motor symptoms in early PD show large inter-individual variation, even in the first year of observation, pointing towards different progression trajectories [4]. Therefore, indicators for disease progression are warranted. Currently, as reported in smaller studies with a small number of parameters, the most important predictors for worse motor progression are age and motor disability at baseline [5]. Recent findings suggest that cardiovascular risk factors contribute to a more severe PD phenotype, but a multimodal approach is lacking [6]. The difficulty 
in identifying early diagnostic criteria for PD depends on the fact that no real biomarker can yet predict illness onset [7]. Therefore, investigating the available laboratory tests and MRI brain basic findings is more feasible and require further research.

In this present study, we assessed the predictors of motor progression of PD over 1 year either clinical (baseline characteristics), neuroimaging or biochemical (serum uric acid, lipid profile, glycated hemoglobin (HbA1c)).

\section{Methods}

In this prospective cohort study, a convenient sample of 75 recruited patients diagnosed with $\mathrm{PD}$ according to International Parkinson and Movement Disorders Society (MDS) diagnostic criteria [8], out of them, 45 patients completed the assessments at baseline, 6 months and after 1 year. Patients were recruited from Movement Disorder Outpatient Clinic at Ain Shams University hospitals in the period between July 2019 and December 2020. Patients with atypical or acquired Parkinsonism or underwent functional brain surgery were excluded.

An informed consent for participation in the study was obtained from all participants following a full explanation of the protocol, that was approved by ethical committee of faculty of medicine, Ain Shams University (FWA 000017585 in 10th of July 2019), according to the Declaration of Helsinki. This study was registered in clinicalTrial.gov, NCT04062279.

Comprehensive medical history, neurological examination, laboratory testing, as well as brain imaging were done initially, followed by assessment scales at baseline, 6 months, and at 1 year. They included motor assessment using MDS-UPDRS during OFF state, Hoehn and Yahr, Schwab and England Activities of Daily Living Scale [9], baseline gait assessment during OFF state by New Freezing of Gait Questionnaire (NFOG-Q) [10], Berg Balance Scale (BBS) [11], 10-Meter Walking Test (10-MWT) [12] and Time Up and Go Test (TUG) [13]; physical activity through International Physical Activity Questionnaire short form (IPAQ) [14], baseline assessment of nonmotor symptoms using the Non-Motor Symptoms Scale (NMSS) [15]; depression assessment by the Arabic version of Beck Depression Inventory (BDI) [16], as well as quality of life testing using the Arabic version of PD questionnaire 39 (PDQ-39) [17]; and cognitive assessment at baseline was done by Mini-Mental State Examination (MMSE) [18]. Levodopa equivalent daily dosage (LEDD) was calculated at baseline as the sum of the daily dose of all dopaminergic agents [19]. Mean difference was calculated as the difference between baseline and followup scores. Percentage change was equal to the change in value divided by the absolute value of the original value, multiplied by 100 .

Serum level of uric acid, lipid profile and $\mathrm{HbA1c}$ were measured initially at baseline. Magnetic resonance imaging (MRI) brain to assess white matter hyperintensities lesions (WMHL) was done using Fazekas scale scoring from Fazekas 0: no or a single punctate WMHL, to Fazekas 3: large confluent lesions [20], Scheltens score [21] for evaluation of WMHL number, size and localization (periventricular, deep white matter, basal ganglion, infratentorial) scoring from $0=$ no abnormalities, to $6=$ confluent lesions [22]. In addition to the whole-group data analysis, comparisons between patients subtypes were performed according to motoric subtypes (TD vs non-TD) [23], male vs female gender, early-onset vs lateonset $\mathrm{PD}$, and mild vs moderate and advanced stages [24].

\section{Statistical analysis}

Data analysis was done by IBM SPSS software package version 25.0 (Armonk, NY: IBM Corp). The normality test was done using Kolmogorov-Smirnov test. Qualitative data were described in frequency and percentage. Quantitative data were described in mean \pm standard deviation. Repeated measures ANOVA (RMANOVA) was used for normally distributed continuous variables of the three-point assessments, while Friedman test was used with post hoc analysis by Wilcoxon tests and Bonferroni correction ( $p$ value is significant if $<0.017$ ) for ordinal variables or not normally distributed continuous ones. Pearson correlation coefficient was used to correlate continuous normally distributed variables, while Spearman correlation was used in case of continuous not normally distributed variables. Mann-Whitney test was used in subgroup analysis. The significance was judged at confidence interval (CI) set to $95 \%$ and statistical significance at $P<0.05$ (except for correlation coefficient in which Bonferroni adjustment was done and calculated according to the following formula $\alpha / m$ where $\alpha$ is the desired overall alpha level and $m$ is the number of hypotheses) [25]. Linear regression was used to investigate the predictors of clinical progression over 1 year. Sample size was calculated by standard formula to give the confidence interval of $95 \%$ and $\pm 5 \%$ margin of error.

\section{Results}

Forty-five patients (34 males, 75.6\%) completed the follow-up assessments up to 1-year. Mean age was $56.46 \pm 9.32$ (ranging from 32 to 78 years old), age of onset was $51.32( \pm 9.67)$ years (ranging $26-74$ years), and mean duration was $4.9( \pm 3.09)$ years (from 0.25 to 14). Mean disease stage Hoehn and Yahr was $2.57 \pm 0.71$ (ranging from 1.5 to 4 ). Twenty-two patients were either 
illiterate (14 patients) or just read and write (8 patients). At baseline, 29 patients (64.4\%) were depressed using BDI ((14 (31.1\%) mild, 8 (17.8\%) moderate and 7 (15.6\%) severe depression). At baseline, 34 (75.5\%) patients were tremor dominant (TD) while 9 (20\%) patients were akinetic rigid and only $2(4.44 \%)$ were indeterminate. Fifteen $(33.33 \%)$ patients were young-onset PD (YOPD) (AOO $<50$ years) and $30(66.67 \%)$ patients were late-onset $\mathrm{PD}$ (LOPD) (AOO $>50$ years). Table 1 and Additional file 1: Table S1 show detailed baseline characteristics of enrolled PD patients including clinical, laboratory and brain imaging. The 1st follow-up (at 6 months) of 35 patients (77.8\%) and 2nd follow-up (at 1 year) of 44 patients (97.8\%) were during the COVID-19 pandemic.

There were significant worsening in MDS-UPDRS total, part III scores, Hoehn and Yahr and Schwab and England ADL scores $(p<0.001)$ at 1 -year follow-up. Also, there was a significant worsening of axial, rigidity $(p<0.001)$, tremor $(p<0.001)$ scores at 1 -year assessment. Also, IPAQ showed highly significant worsening at 1 -year follow-up $(p<0.001)$ (Table 2, Fig. 1).

According to Hoehn and Yahr staging, 34 (75.6\%) patients had early (mild) PD (stages 1-2.5) at baseline vs $18(40.9 \%)$ patients at 1-year follow-up, while 5 (11.1\%) had advanced PD (stages 4-5) at baseline vs 14 $(31.8 \%)$ at 1-year follow-up ( $p$ 0.004).

It was noticed that the motor progression was more obvious in the second half of follow-up year especially in MDS-UPDRS part I, III, and MDS-UPDRS subscores, as well as Hoehn and Yahr staging (Table 2 and Fig. 1). The mean rate of motor progression at 1 year was 26.93\% for MDS-UPDRS III, 18.78\% for Hoehn and Yahr and $17.19 \%$ of Schwab and England ADL in the Off state (Additional file 1: Table S2).

Comparing 34 patients with early/mild PD (Hoehn and $\mathrm{Yahr} \leq 2.5)$ vs 11 patients with moderate and advanced PD stages (Hoehn and Yahr 3, 4,5), baseline scores of MDS-UPDRS total, part II and part III were higher in advanced and moderate stages $(p<0.001)$. Moreover, NFOG-Q, TUG, IPAQ and Schwab and England ADL baseline scores were also higher in advanced and moderate stages $(p<0.001)$. On the other hand, disease progression was not significant in all parameters between early and advanced stages except for Schwab and England ADL $(p=0.01)$ (Additional file 1: Table S3).

Table 1 Baseline demographic data and clinical characteristics of PD patients

\begin{tabular}{|c|c|c|}
\hline & Mean (SD)/frequency (\%) & Range \\
\hline Age (years) & $56.45(9.31)$ & $32-78$ \\
\hline \multicolumn{3}{|l|}{ Gender } \\
\hline Male & $34(75.6 \%)$ & \\
\hline Female & $11(24.4 \%)$ & \\
\hline AOO (years) & $51.32(9.67)$ & $26-74$ \\
\hline DOI (years) & $4.90(3.1)$ & $0.25-14$ \\
\hline MDS-UPDRS Total score OFF & $81.75(33.02)$ & $29-176$ \\
\hline MDS-UPDRS-I & $15.25(7.29)$ & $1-35$ \\
\hline MDS-UPDRS-\| & $18.48(10.19)$ & $0-44$ \\
\hline MDS-UPDRS-III OFF & $47.89(19.82)$ & $13-101$ \\
\hline Hoehn and Yahr OFF & $2.53(0.69)$ & $1.5-4$ \\
\hline Schwab and England ADL OFF & $76.14(13.16)$ & $30-90$ \\
\hline TUG OFF & $19.21(16.31)$ & $7.86-82.80$ \\
\hline (10-MWT) Comfortable speed OFF (m/s) & $0.77(0.33)$ & $0.10-1.29$ \\
\hline (10-MWT) Maximum speed OFF (m/s) & $1.031(0.43)$ & $0.12-1.75$ \\
\hline BBS OFF & $45.77(9.91)$ & $14-56$ \\
\hline NFOG-Q OFF & $10.57(10.10)$ & $0-29$ \\
\hline IPAQ & $2058.77(821.20)$ & $636-3942$ \\
\hline MMSE & $26.45(3.17)$ & $16-30$ \\
\hline NMSS total score & $61.57(44.19)$ & $4-205$ \\
\hline BDI & 17.61 (9.87) & $0-51$ \\
\hline PDQ-39 & 37.08 (18.68) & $2.6-76.7$ \\
\hline
\end{tabular}

$A O O$ age of onset, $D O I$ duration of illness, MDS-UPDRS Movement Disorder Society—Unified Parkinson's Disease Rating Scale, ADL activities of daily living, TUG Time Up and Go Test, 10-MWT 10-Meter Walking Test, BBS Berg Balance Scale, NFOG-Q New Freezing of Gait Questionnaire, IPAQ International Physical Activity Questionnaire, MMSE Mini-Mental State Examination, NMSS Non-Motor Symptoms Scale, BDI Beck Depression Inventory, PDQ_39 Parkinson's Disease Questionnaire-39 
Table 2 Progression of motor and physical activity over 6-month and 1-year follow-up

\begin{tabular}{|c|c|c|c|c|c|c|c|}
\hline & $\begin{array}{l}\text { Baseline } \\
\text { Mean (SD) }\end{array}$ & $\begin{array}{l}\text { 6-month FU } \\
\text { Mean (SD) }\end{array}$ & $\begin{array}{l}\text { 1-year FU } \\
\text { Mean (SD) }\end{array}$ & $\begin{array}{l}\text { RMANOVA } \\
p\end{array}$ & $\begin{array}{l}\text { Baseline } \\
\text { versus } \\
6 \text { months }\end{array}$ & $\begin{array}{l}6 \text { months } \\
\text { versus } \\
1 \text { year }\end{array}$ & $\begin{array}{l}\text { Baseline } \\
\text { versus } \\
1 \text { year }\end{array}$ \\
\hline MDS-UPDRS total score OFF & $81.75(33.02)$ & $86.50(29.66)$ & $105.86(33.21)$ & $<0.001^{*}$ & $0.009 *$ & $<0.001 *$ & $<0.001^{*}$ \\
\hline MDS-UPDRS-I & $15.25(7.29)$ & $16.25(6.24)$ & $21.34(6.01)$ & $<0.001^{*}$ & 0.149 & $<0.001 *$ & $<0.001^{*}$ \\
\hline MDS-UPDRS-II & $18.48(10.19)$ & $20.70(8.62)$ & $25.02(9.14)$ & $<0.001 *$ & $0.013^{*}$ & $<0.001 *$ & $<0.001^{*}$ \\
\hline MDS-UPDRS-III OFF & $47.89(19.82)$ & $49.25(18.95)$ & $59.52(21.65)$ & $<0.001 *$ & 0.288 & $<0.001 *$ & $<0.001^{*}$ \\
\hline Tremor OFF & $12.80(7.47)$ & $13.00(7.56)$ & $14.52(8.03)$ & $<0.001^{*}$ & 1 & $0.001 *$ & $0.002^{*}$ \\
\hline Bradykinesia OFF & $15.93(7.57)$ & $16.57(7.08)$ & $20.93(7.68)$ & $<0.001 *$ & 0.356 & $<0.001 *$ & $<0.001^{*}$ \\
\hline Rigidity OFF & $8.91(4.15)$ & $9.09(3.68)$ & $10.66(3.95)$ & $<0.001^{*}$ & 1 & $<0.001 *$ & $<0.001 *$ \\
\hline Axial OFFa & 13.93(6.88) & $14.70(6.13)$ & $18.23(6.86)$ & $<0.001 *$ & 0.014 & $<0.001 *$ & $<0.001^{*}$ \\
\hline PIGD OFF & $8.55(5.72)$ & $8.84(5.26)$ & $10.70(5.54)$ & $<0.001^{*}$ & 0.193 & $<0.001^{*}$ & $<0.001^{*}$ \\
\hline Motor complication total score ${ }^{a}$ & $4.93(4.48)$ & $5.89(4.18)$ & $6.91(4.23)$ & $<0.001^{*}$ & 0.004 & $<0.001 *$ & $<0.001 *$ \\
\hline Hoehn and Yahr OFFa & $2.53(0.69)$ & $2.63(0.75)$ & $3.00(0.87)$ & $<0.001^{*}$ & 0.024 & $<0.001 *$ & $<0.001 *$ \\
\hline Schwab and England ADL OFF' & $76.14(13.16)$ & $71.14(16.46)$ & $63.64(16.72)$ & $<0.001^{*}$ & $<0.001$ & $<0.001^{*}$ & $<0.001^{*}$ \\
\hline IPAQ & $2058.77(821.20)$ & $1622.21(779.69)$ & $1186.73(605.78)$ & $<0.001^{*}$ & $<0.001^{*}$ & $<0.001^{*}$ & $<0.001 *$ \\
\hline
\end{tabular}

Bold values are significant

MDS-UPDRS Movement Disorder Society—Unified Parkinson's Disease Rating Scale, PIGD postural instability and gait disorder, ADL activities of daily living, IPAQ International Physical Activity Questionnaire, RMANOVA repeated measures ANOVA

${ }^{a}$ Friedman's test was used and post hoc analysis was done by Wilcoxon tests with Bonferroni correction ( $p$ value is significant if $<0.017$ )

* $p$ value is significant if $<0.05$

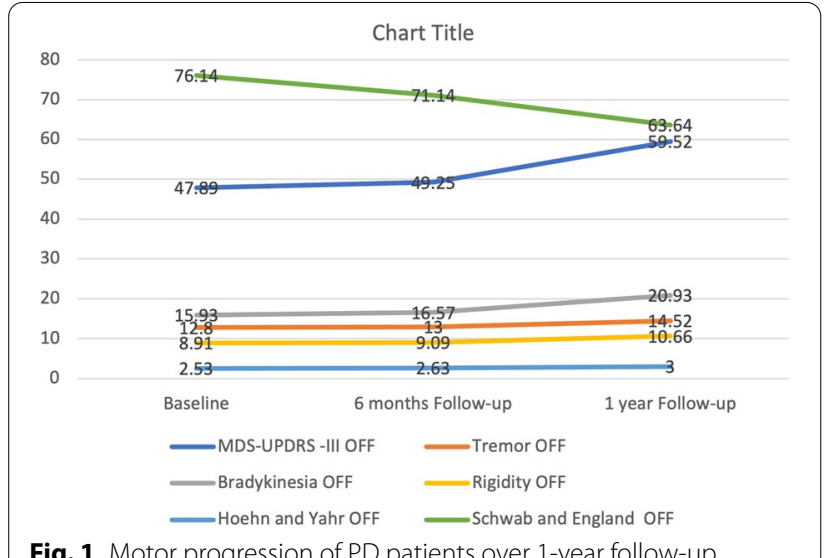

Fig. 1 Motor progression of PD patients over 1-year follow-up

Male and female patients were matched regarding age, disease duration and severity. Compared to males, female patients had significantly higher NFOG-Q $(p=0.03)$ and PIGD $(p=0.03)$, prolonged TUG $(p=0.03)$, slower 10-MWT comfortable $(p=0.02)$ and maximum speed $(p=0.03)$, and higher serum HDL level $(p=0.02)$. On the other hand, disease progression was not significant between males or females in all parameters (Additional file 1: Table S4).

Patients with non-TD PD subtype presented earlier in life compared to TD PD $(p=0.046)$ with longer DOI $(p=0.039)$. Expectedly, the MDS-UPDRS tremor subscore was significantly higher in TD PD $(p<0.001)$ while PIGD was higher in non-TD $(p=0.005)$. Patients with TD PD were significantly faster than the non-TD PD subtype $(p=0.039)$, with lower freezing of gait scores $(p=0.001)$. and better quality of life $(p=0.017)$ compared to non-TD PD. Regarding disease progression, only Hoehn and Yahr and IPAQ showed significantly worse progression among patients with the non-TD type ( $p=0.040$ and 0.012 , respectively) (Additional file 1: Table S5).

Comparing EOPD (15 patients) with LOPD (30 patients), the former had significantly shorter disease duration $(p=0.040)$, higher HbA1C $(p=0.01)$ and higher Scheltens total scores $(p=0.04)$, respectively. Yet, there was no significant difference regarding clinical progression between the two groups (Additional file 1: Table S6).

One-year progression of Hoehn and Yahr and Schwab and England ADL were significantly correlated to disease duration ( $\mathrm{rho}=0.367, p=0.014$; and rho $=0.337$, $p=0.025$, respectively). Progression of Hoehn and Yahr was correlated to baseline TUG $(\mathrm{rho}=0.319, p=0.035)$. Schwab and England ADL progression was correlated to baseline of MDS-UPDRS total score $(\mathrm{rho}=0.478$, $p=0.001)$, PIGD (rho $=0.591, p<0.001)$, NFOG-Q baseline (rho $=0.498, p=0.001$ ), TUG (rho $=0.565$, $p=0.001)$, MDS-UPDRS part III (rho $=0.350$, $p=0.020)$, and Hoehn and Yahr (rho $=401, p=0.007)$ (Table 3).These correlations were non-significant after Bonferroni correction $(p<0.003)$ except for Schwab and England ADL progression correlation with baseline 


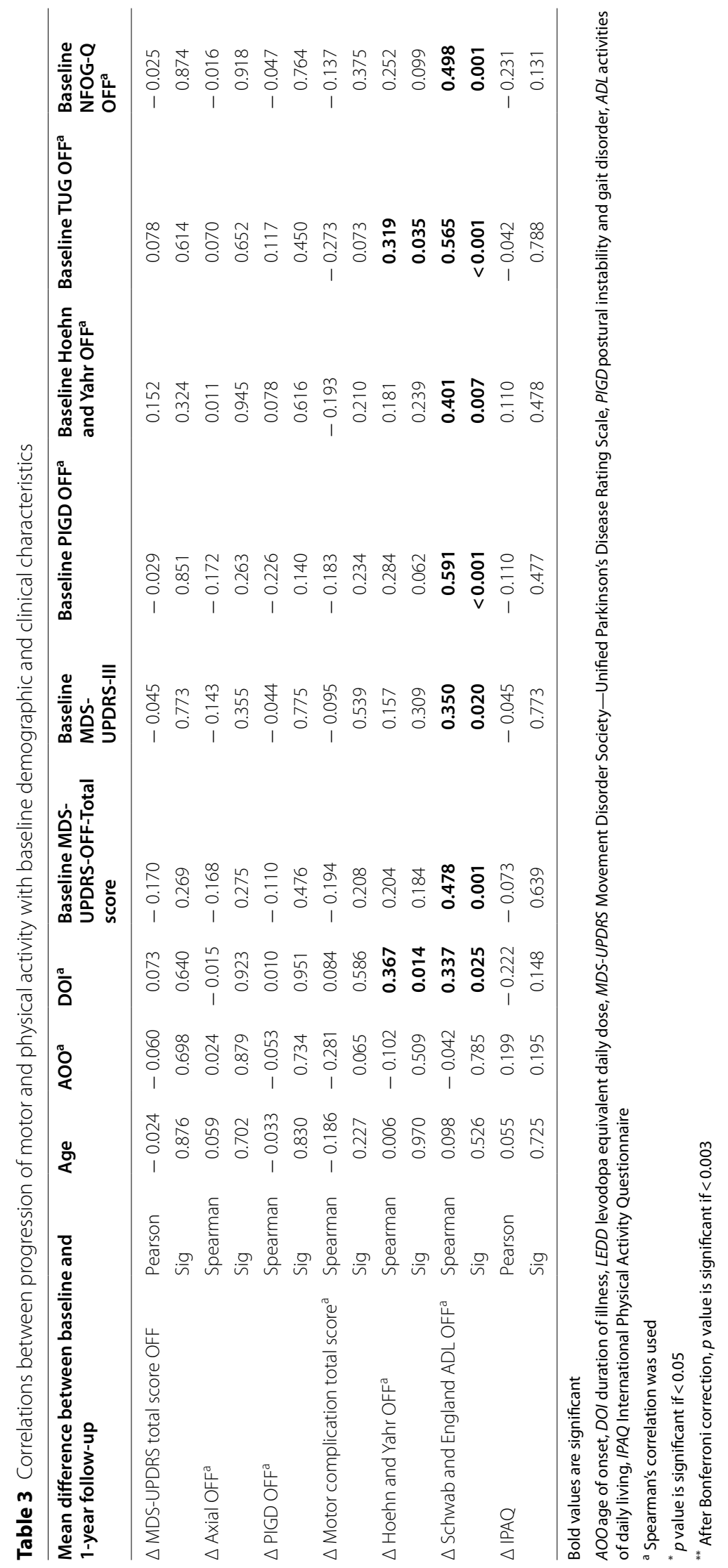


MDS-UPDRS total score, PIGD, TUG and NFOG-Q (Table 3, Additional file 1: Table S7).

One-year progression of MDS-UPDRS total score, PIGD, and axial were inversely correlated to baseline NMSS total score $(r=-0.376, p=0.012$; rho $=-0.371$, $p=0.013 ; \quad$ rho $=-0.392, \quad p=0.009$, respectively). Schwab and England ADL progression was directly correlated to baseline of PDQ-39, BDI and NMSS total score $(\mathrm{rho}=0.426, p=0.004$; rho $=0.302, p=0.046$ and rho $=302, p=0.046$, respectively) and inversely correlated to baseline MMSE (rho $=-399, p=0.007)$. Progression of motor complications and IPAQ were correlated to baseline of IPAQ (rho $=0.379, p=0.011$; rho $=675, p<0.001$, respectively). Progression of axial and motor complications were inversely correlated to baseline BDI (rho $=-298, p=0.49$; rho $=-360$, $p=0.017$, respectively). These correlations were nonsignificant after Bonferroni correction $(p<0.003)$ except physical activity worsening with baseline physical activity $(p<0.001)$ (Table 4).

PIGD progression was directly correlated to LDL level $(\mathrm{rho}=322, p=0.043)$. Motor complications progression was moderately correlated to triglycerides level $($ rho $=0.313, p=0.049)$. These correlations were nonsignificant after Bonferroni correction. No other significant correlations with other test and MRI findings were detected (Table 5).
Predictors of clinical progression were assessed by linear regression from the following variables: DOI, total MDS-UPDRS, MDS-UPDRS III, tremor subscore, PIGD subscore, Hoehn and Yahr stage, LEDD, total non-motor scale score, BDI, TUG, (10-MWT) comfortable speed, NFOG-Q, Fazekas total score, uric acid. Using linear regression, there was no predictors of clinical progression among the variables used.

\section{Discussion}

The rate of progression of PD differs widely among individual patients [26]. Therefore, many studies are required to determine progression of PD and its predictors. The current study investigated disease progression within 1 year and its potential clinical and laboratory predictors in a cohort of patients with PD in an under-investigated population. Despite of the short follow-up of the current study, identifying disease progression within 1 year enables comparing annual progression with other populations. Additionally, the annual motor progression was suggested as a predictor of long-term disease progression [27].

The current study confirmed other previous observations. Significant motor progression from baseline was first detected at 1-year follow-up consistent with previous studies [28]. Furthermore, variability of motor progression among patients with PD was also observed similar to previous studies $[2,28]$.

Table 4 Correlations between progression of motor and physical activity with baseline cognitive, NMSS and quality of life

\begin{tabular}{|c|c|c|c|c|c|c|c|}
\hline \multicolumn{2}{|c|}{$\begin{array}{l}\text { Mean difference between baseline and } \\
1 \text {-year follow-up }\end{array}$} & \multirow{2}{*}{$\begin{array}{c}\text { Baseline MMSE } \\
0.046\end{array}$} & \multirow{2}{*}{$\begin{array}{l}\begin{array}{l}\text { Baseline } \\
\text { NMSS total } \\
\text { score }\end{array} \\
-0.376^{*}\end{array}$} & \multirow{2}{*}{$\begin{array}{l}\text { Baseline BDI } \\
-0.220\end{array}$} & \multirow{2}{*}{$\begin{array}{l}\text { Baseline PDQ-39 } \\
-0.211\end{array}$} & \multirow{2}{*}{$\begin{array}{c}\text { Baseline IPAQ } \\
0.125\end{array}$} & \multirow{2}{*}{$\begin{array}{l}\text { Baseline LEDD } \\
-0.133\end{array}$} \\
\hline$\triangle$ MDS-UPDRS total score OFF & Pearson & & & & & & \\
\hline & Sig & 0.765 & 0.012 & 0.152 & 0.170 & 0.420 & 0.391 \\
\hline \multirow[t]{2}{*}{$\triangle$ Axial OFF } & Spearman & -0.039 & -0.392 & -0.298 & -0.298 & 0.231 & -0.125 \\
\hline & Sig & 0.802 & 0.009 & 0.049 & 0.050 & 0.131 & 0.418 \\
\hline \multirow[t]{2}{*}{$\triangle \mathrm{PIGD} \mathrm{OFF}^{\mathrm{a}}$} & Spearman & -0.102 & -0.371 & -0.289 & -0.222 & 0.152 & -0.115 \\
\hline & Sig & 0.510 & 0.013 & 0.057 & 0.148 & 0.325 & 0.459 \\
\hline \multirow{2}{*}{ 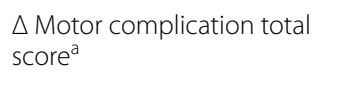 } & Spearman & 0.002 & -0.225 & -0.360 & -0.116 & 0.379 & 0.006 \\
\hline & Sig & 0.991 & 0.141 & 0.017 & 0.454 & 0.011 & 0.971 \\
\hline \multirow[t]{2}{*}{$\Delta$ Hoehn and Yahr OFF' } & Spearman & -0.048 & 0.118 & 0.172 & 0.186 & -0.052 & 0.171 \\
\hline & Sig & 0.756 & 0.444 & 0.264 & 0.227 & 0.736 & 0.268 \\
\hline \multirow{2}{*}{$\begin{array}{l}\triangle \text { Schwab and England ADL } \\
\text { OFF }^{\mathrm{a}}\end{array}$} & Spearman & -0.399 & 0.302 & 0.302 & 0.426 & -0.207 & 0.259 \\
\hline & Sig & 0.007 & 0.046 & 0.046 & 0.004 & 0.178 & 0.089 \\
\hline \multirow[t]{2}{*}{$\triangle \mathrm{IPAQ}$} & Pearson & 0.002 & -0.028 & -0.228 & -0.075 & $0.675^{* *}$ & -0.114 \\
\hline & Sig & 0.988 & 0.855 & 0.137 & 0.631 & $<0.001$ & 0.461 \\
\hline
\end{tabular}

Bold values are significant

MDS-UPDRS Movement Disorder Society—Unified Parkinson's Disease Rating Scale PIGD postural instability and gait disorder, ADL activities of daily living, IPAQ International Physical Activity Questionnaire, MMSE Mini-Mental State Examination, NMSS Non-Motor Symptoms Scale, BDI Beck Depression Inventory, PDQ_39 Parkinson's Disease Questionnaire-39

${ }^{*} p$ value is significant if $<0.05$

${ }^{*}$ After Bonferroni correction, $p$ value is significant if $<0.003$ 
Table 5 Correlations between progression of motor and physical activity with laboratory and imaging characteristics

\begin{tabular}{|c|c|c|c|c|c|c|c|c|c|}
\hline \multicolumn{2}{|c|}{$\begin{array}{l}\text { Absolute change } \\
\text { between baseline and } \\
\text { 1-year follow-up }\end{array}$} & \multirow{2}{*}{$\begin{array}{c}\mathrm{HbA} 1 \mathrm{c} \%^{\mathrm{a}} \\
0.027\end{array}$} & \multirow{2}{*}{$\begin{array}{c}\begin{array}{l}\text { Uric acid } \\
\text { (mg/dL) }\end{array} \\
0.102\end{array}$} & \multirow{2}{*}{$\begin{array}{c}\begin{array}{l}\text { Cholesterol } \\
\text { (mg/dL) }\end{array} \\
0.226\end{array}$} & \multirow{2}{*}{$\begin{array}{l}\text { Triglycerides } \\
\text { mg/dL) }\end{array}$} & \multirow{2}{*}{$\begin{array}{c}\mathrm{LDL}(\mathrm{mg} / \mathrm{dL}) \\
0.295\end{array}$} & \multirow{2}{*}{$\begin{array}{l}\mathrm{HDL}(\mathrm{mg} / \mathrm{dL})^{\mathrm{a}} \\
-0.113\end{array}$} & \multirow{2}{*}{$\begin{array}{l}\begin{array}{l}\text { Fazekas } \\
\text { total }^{\mathrm{a}}\end{array} \\
-0.084\end{array}$} & \multirow{2}{*}{$\begin{array}{l}\begin{array}{l}\text { Scheltens } \\
\text { total }^{\mathbf{a}}\end{array} \\
-0.003\end{array}$} \\
\hline$\triangle$ MDS-UPDRS & Pearson & & & & & & & & \\
\hline & Sig & 0.865 & 0.532 & 0.161 & 0.731 & 0.065 & 0.498 & 0.592 & 0.985 \\
\hline \multirow[t]{2}{*}{$\triangle$ Axial OFFa } & Spearman & 0.000 & 0.209 & 0.133 & 0.056 & 0.256 & -0.064 & 0.002 & 0.028 \\
\hline & Sig & 0.998 & 0.196 & 0.415 & 0.733 & 0.110 & 0.701 & 0.990 & 0.859 \\
\hline \multirow[t]{2}{*}{$\triangle P I G D O F F^{a}$} & Spearman & -0.194 & 0.276 & 0.171 & 0.230 & 0.322 & -0.068 & -0.089 & 0.004 \\
\hline & Sig & 0.224 & 0.084 & 0.292 & 0.154 & 0.043 & 0.685 & 0.571 & 0.978 \\
\hline \multirow{2}{*}{$\begin{array}{l}\triangle \text { Motor com- } \\
\text { plication total } \\
\text { score }^{\text {a }}\end{array}$} & Spearman & -0.217 & -0.111 & 0.065 & 0.313 & 0.013 & -0.050 & -0.146 & -0.059 \\
\hline & Sig & 0.172 & 0.495 & 0.691 & 0.049 & 0.939 & 0.766 & 0.350 & 0.704 \\
\hline \multirow{2}{*}{$\begin{array}{l}\triangle \text { Hoehn and } \\
\text { Yahr OFF }\end{array}$} & Spearman & 0.198 & 0.148 & 0.122 & -0.151 & 0.139 & -0.005 & 0.041 & 0.105 \\
\hline & Sig & 0.215 & 0.362 & 0.452 & 0.351 & 0.393 & 0.976 & 0.795 & 0.496 \\
\hline \multirow{2}{*}{$\begin{array}{l}\triangle \text { Schwab and } \\
\text { England ADL } \\
\text { OFF }^{\mathrm{a}}\end{array}$} & Spearman & 0.113 & 0.020 & -0.113 & -0.102 & -0.086 & 0.051 & -0.019 & 0.096 \\
\hline & Sig & 0.482 & 0.902 & 0.487 & 0.532 & 0.598 & 0.760 & 0.901 & 0.537 \\
\hline \multirow[t]{2}{*}{$\triangle \mathrm{IPAQ}$} & Pearson & 0.066 & 0.009 & -0.014 & 0.027 & 0.018 & 0.023 & 0.086 & -0.136 \\
\hline & Sig & 0.683 & 0.958 & 0.933 & 0.867 & 0.912 & 0.891 & 0.582 & 0.379 \\
\hline
\end{tabular}

Bold values are significant

$L D L$ low-density lipoproteins, $H D L=$ high-density lipoproteins, MDS-UPDRS Movement Disorder Society-Unified Parkinson's Disease Rating Scale PIGD= postural instability and gait disorder, $A D L$ activities of daily living, IPAQ International Physical Activity Questionnaire

* $p$ value is significant if $<0.05$

${ }^{* *}$ After Bonferroni correction, $p$ value is significant if $<0.003$

Remarkably, the current study showed a high rate of motor progression compared to previous studies $[2,27$, 29-31]. The mean difference of MDS-UPDRS III was -11.83 , while the rate of progression was $26.93 \%$ within 1 year. Schrag and his colleagues showed mean annual progression rates of motor impairment and disability ranged from 2.4 to $7.4 \%$. This could be explained by the lower age of onset and severity of the current cohort, as motor progression decrease with advancing disease [2]. Moreover, the presence of medical comorbidities in about half of recruited patients (46.7\%) also could explain this higher rate of progression [32].

Remarkably, most of the 1-year follow-ups in the current study were performed during the COVID-19 pandemic. Therefore, these special circumstances may be another important factor for that fast progression which showed agreement with a study done in 2021 in India. The study showed that the most common motor symptom to worsen was motor slowness which was seen in $69.2 \%$ of cases followed by tremors, rigidity and gait freezing [33]. Additionally, patients with PD reported worse mental health, quality of life, and physical inactivity during this pandemic [34]. Patients with PD are more vulnerable to recent stressors as a dopamine depleted brain leads to reduced coping mechanisms to stress. Moreover, chronic stress may lead to increased striatonigral dopaminergic neuron loss via oxidative damage to the cell membrane, inflammatory and regulatory T-cell dysfunction, thus leading to worsening of motor symptoms, also it leads to greater activation of locus coeruleus-noradrenergic pathways and may worsen the resting tremor in PD [35, 36].

In addition to individual variability, motor progression of PD is variable between patients and disease subgroups. The Schwab and England ADL progression was more significant in patients with advanced stages of PD compared to wild PD, and this was consistent with previous studies which reported that mobility, activities of daily living, communication, and stigma were more progressed in advanced PD patients [37].

There was no significant difference in progression in either males or females. Similarly, Reinoso and his colleagues described a similar motor progression in males and females over 1 year, but significant differences were detected in longer periods [30]. More recently, another study with a large cohort confirmed similar rate of progression of males and females [38]. Inconsistently, Chahine and his colleagues reported male gender as a predictor for motor progression [27] and a recent metaanalysis suggested that females progress more slowly regarding ADL impairment and develop dyskinesia early [39], implying the need for further studies. 
Minimal differences in motor progression were detected between TD and non-TD subtypes including disease severity (Hoehn and Yahr) and physical activity, similar to previous studies. Reinoso and his colleagues reported higher motor progression with akinetic rigid subtype [30]. Similarly, another study reported subtle differences between TD and PIGD subtypes during 4-year observation, despite more severe manifestations at baseline [40]. A previous study showed more rapid motor progression with PIGD versus TD, but without predictive value [5].

Comparing early-onset and late-onset PD at baseline showed no significant difference in motor progression. This is inconsistent with a previous study that found slower progression in EOPD and suggests that the preclinical interval in this group is longer [41]. Another study showed a similar progression rate in the first 4 years post-diagnosis, followed by more rapid progression in the older group [30]. Younger age and shorter duration of our cohort, small sample size and short duration of follow-up may explain these differences.

Predictors of motor progression were variable among previous studies and included male gender, higher baseline motor score, male sex, and increased age, akinetic rigid subtype, cognitive impairment, nondopaminergic symptoms in addition to genetic factors and CSF biomarkers [3, 30, 42]. The current study showed significant associations between motor progression over 1 year and baseline scores, especially Schwab and England ADL progression and gait parameters. However, this study did not detect significant predictors of motor progression over 1 year that could be explained by small sample size, short duration of follow-up and different subtypes of PD included in this cohort. Furthermore, this was consistent with previous studies which showed conflicting results in detecting prognostic factors of PD progression due to short duration of follow-up and variable methods used [43]. Therefore, longer follow-up and larger cohorts are warranted.

A recent population-based study showed the influence of lifestyle factors including physical activity on PD progression [44]. Therefore, the role of physical activity was investigated in this study and showed significant association with worsening of Schwab and England ADL and physical activity, denoting its potential prognostic value and the importance of improving the lifestyle of people with PD [45]. Moreover, baseline NMS showed associated with progression of total MDS-UPDRS, PIGD, Schwab and England ADL and axial scores. Similarly, Ayala and his colleagues reported the NMS as a predictor of disease progression over 3 years [46]. Remarkably, the current cohort is characterized by younger age and age of onset, shorter disease duration, better baseline cognition, that affect study outcome and interpretation.

Previous studies linked WMH severity or volume to motor, gait and cognitive progression of $\mathrm{PD}$, implying its usefulness as a prognostic marker [47-49]. The current study showed variability of WMH in size in agreement with previous studies [50], yet these changes could not predict progression over 1 year. Inconsistency with other studies may be attributed to different methodology, a small number of patients, using low-resolution MRI, short follow-up and low WMH load of most of the recruited patients. Therefore, further MRI studies are warranted to evaluate short prognostic values of WMH.

Baseline lipid profile was not correlated to motor progression of PD in the current study in agreement with previous studies, which failed to find a significant linkage between lipid profile abnormalities and PD progression $[51,52]$. Triglyceride level was correlated to progression of motor complications in this study, which is inconsistent with previous studies demonstrating no significant association between triglycerides and motor or cognitive progression [51]. However, previous studies revealed controversy regarding the association between lipid profile and PD progression [53]. Serum uric acid was not correlated to PD progression in this cohort, in contrast to previous studies which reported that low levels of serum uric acid associated with a high risk of PD progression and worsening of UPDRS scores [54, 55]. Moreover, HbA1c was not correlated to PD progression in this study in agreement with the uncertainty about the role of HbA1c to predict PD progression in previous studies [56]. Younger age, shorter duration of our cohort, small sample size and short duration of follow-up should be considered when interpreting the current findings.

Although investigating the baseline predictors (clinical and biochemical) of PD in a follow-up study over 1 year is important, larger sample size with a longer duration is required for more convenient analysis. Furthermore, including more biochemical biomarkers and advanced neuroimaging are required. Moreover, the COVID-19 pandemic impacted the recruitment of the patients, in addition to its possible effect on disease progression. Also, assessment of therapy adherence among PD patients is required especially during COVID-19 pandemic.

\section{Conclusion}

The current study demonstrated the motor progression over 1 year in an under-investigated population that showed high annual worsening. It showed significant associations between motor progression over 1 year and baseline scores, especially Schwab and England ADL 
progression, and gait parameters, but without predictive values. Remarkably, it highlighted the progression of physical activity. However, further longitudinal studies with a larger number are warranted to detect and confirm predictors for short-term progression.

\section{Abbreviations}

ADL: Activities of daily living; AOO: Age of onset; BBS: Berg Balance Scale; BDI: Beck Depression Inventory; COVID-19: Coronavirus disease of 2019; CSF: Cerebrospinal fluid; DOI: Duration of illness; EOPD: Early-onset PD; HDL: High-density lipoprotein; IPAQ: International Physical Activity Questionnaire; LDL: Low-density lipoprotein; LEDD: Levodopa equivalent daily dose; LOPD: Late-onset PD; MDS: Movement Disorder Society; MMSE: Mini-Mental State Examination; MRI: Magnetic resonance imaging; NFOG-Q: New Freezing Of Gait Questionnaire; NMSS: Non-Motor Symptoms Scale; PD: Parkinson's disease; PDQ-39: PD Questionnaire-39; QoL: Quality of life; RBD: Rapid eye movement sleep behavior disorder; RMANOVA: Repeated measures ANOVA; TD:Tremor dominant; TUG: Time Up and Go Test; UPDRS: Unified Parkinson's Disease Rating Scale; WMHL: White matter hyperintensities lesions; 10-MWT: 10-Meter Walking Test.

\section{Supplementary Information}

The online version contains supplementary material available at https://doi. org/10.1186/s41983-022-00445-1.

Additional file 1: Table S1. Education, comorbidities, laboratory results, brain imaging, and Hoehn and Yahr stagging of PD patients at baseline. Table S2. Percentage change of motor and physical activity over 6 months and 1-year follow-up. Table S3. Comparison between mild PD vs advanced-moderate PD regarding baseline characteristics and motor progression. Table S4. Gender comparison regarding baseline characteristics and motor progression. Table S5. Comparison between Tremor Dominant and non-TD patients regarding baseline characteristics and motor progression. Table S6. Comparison between late onset vs early onset PD regarding baseline characteristics and motor progression. Table S7. Correlations between progression of motor subscores with baseline demographic, clinical, Lab and imaging characteristics.

\section{Acknowledgements}

Not applicable.

\section{Authors' contributions}

A.H.: data collection, analysis, interpretation of data, drafted, reviewed, and approved the manuscript. M.S., M.E., and A.G.: design, analysis, interpretation of data, reviewed and approved the manuscript. A.S. and E.H.: conception, design, analysis, interpretation of data, drafted, reviewed and approved the manuscript. All authors have read and approved the final manuscript.

\section{Funding}

Not applicable.

\section{Availability of data and materials}

All data generated or analyzed during the current study are included in this published article (and its Additional information files).

\section{Declarations}

\section{Ethics approval and consent to participate}

The study protocol was approved by Ain Shams University, Faculty of Medicine Research Ethical Committee FWA 000017585, 10th July 2019. Written informed consent was obtained from the patients participating in the study, or their first-degree relatives if the patient was unable to provide consent, after informing them about the study rationale and their right to withdraw from the study at any time without any consequences.

\section{Consent for publication}

Not applicable.

\section{Competing interests}

All authors declare that they do not have any competing interests.

\section{Author details}

${ }^{1}$ Department of Neurology, Faculty of Medicine, Ain Shams University, 168 Elnozha St, Saint Fatima Square, Heliopolis, Cairo, Egypt. ${ }^{2}$ Institute of Global Health and Human Ecology (I-GHHE), The American University in Cairo, Cairo, Egypt. ${ }^{3}$ Faculty of Medicine, Al-Mansoura University, Mansoura, Egypt.

Received: 8 November 2021 Accepted: 1 January 2022

Published online: 15 January 2022

\section{References}

1. Lee A, Gilbert RM. Epidemiology of Parkinson disease. Neurol Clin. 2016;34(4):955-65.

2. Schrag A, Dodel R, Spottke A, Bornschein B, Siebert U, Quinn NP. Rate of clinical progression in Parkinson's disease. A prospective study. Mov Disord. 2007;22(7):938-45.

3. Latourelle JC, Beste MT, Hadzi TC, Miller RE, Oppenheim JN, Valko MP, et al. Large-scale identification of clinical and genetic predictors of motor progression in patients with newly diagnosed Parkinson's disease: a longitudinal cohort study and validation. Lancet Neurol. 2017;16(11):908-16.

4. Simuni T, Siderowf A, Lasch S, Coffey CS, Caspell-Garcia C, Jennings D, et al. Longitudinal change of clinical and biological measures in early Parkinson's disease: Parkinson's progression markers initiative cohort. Mov Disord. 2018;33(5):771-82.

5. Vu TC, Nutt JG, Holford NH. Progression of motor and nonmotor features of Parkinson's disease and their response to treatment. Br J Clin Pharmacol. 2012;74(2):267-83.

6. Malek N, Lawton MA, Swallow DM, Grosset KA, Marrinan SL, Bajaj N, et al. Vascular disease and vascular risk factors in relation to motor features and cognition in early Parkinson's disease. Mov Disord. 2016;31(10):1518-26.

7. Cova I, Priori A. Diagnostic biomarkers for Parkinson's disease at a glance: where are we? J Neural Transm (Vienna). 2018;125(10):1417-32.

8. Postuma RB, Berg D, Stern M, Poewe W, Olanow CW, Oertel W, et al MDS clinical diagnostic criteria for Parkinson's disease. Mov Disord. 2015;30(12):1591-601.

9. Goetz CG, Tilley BC, Shaftman SR, Stebbins GT, Fahn S, Martinez-Martin $P$, et al. Movement Disorder Society-sponsored revision of the Unified Parkinson's Disease Rating Scale (MDS-UPDRS): scale presentation and clinimetric testing results. Mov Disord. 2008;23(15):2129-70.

10. Giladi N, Tal J, Azulay T, Rascol O, Brooks DJ, Melamed E, et al. Validation of the freezing of gait questionnaire in patients with Parkinson's disease Mov Disord. 2009;24(5):655-61.

11. Berg KO, Wood-Dauphinee SL, Williams JI, Maki B. Measuring balance in the elderly: validation of an instrument. Can J Public Health. 1992;83(Suppl 2):S7-11.

12. Wolf SL, Catlin PA, Gage K, Gurucharri K, Robertson R, Stephen K. Establishing the reliability and validity of measurements of walking time using the Emory Functional Ambulation Profile. Phys Therapy. 1999;79(12):1122-33.

13. Bohannon RW. Reference values for the timed up and go test: a descriptive meta-analysis. J Geriatr Phys Therapy. 2006;29(2):64-8.

14. Craig CL, Marshall AL, Sjöström M, Bauman AE, Booth ML, Ainsworth BE, et al. International physical activity questionnaire: 12 -country reliability and validity. Med Sci Sports Exerc. 2003;35(8):1381-95.

15. Chaudhuri KR, Martinez-Martin P, Brown RG, Sethi K, Stocchi F, Odin P, et al. The metric properties of a novel non-motor symptoms scale for Parkinson's disease: results from an international pilot study. Mov Disord. 2007;22(13):1901-11

16. Fawzi MH, Fawzi MM, Abu-Hindi W. Arabic version of the Major Depression Inventory as a diagnostic tool: reliability and concurrent and discriminant validity. East Mediterr Health J. 2012;18(4):304-10.

17. Shalash AS, Hamid E, Elrassas HH, Bedair AS, Abushouk Al, Khamis M, et al. Non-motor symptoms as predictors of quality of life in Egyptian patients with Parkinson's disease: a cross-sectional study using a culturally 
adapted 39-item Parkinson's Disease Questionnaire. Front Neurol. 2018;9:357.

18. Tuijl JP, Scholte EM, de Craen AJ, van der Mast RC. Screening for cognitive impairment in older general hospital patients: comparison of the Six-Item Cognitive Impairment Test with the Mini-Mental State Examination. Int J Geriatr Psychiatry. 2012;27(7):755-62.

19. Shalash A, Alexoudi A, Knudsen K, Volkmann J, Mehdorn M, Deuschl G. The impact of age and disease duration on the long-term outcome of neurostimulation of the subthalamic nucleus. Parkinsonism Relat Disord. 2014;20(1):47-52.

20. Fazekas F, Chawluk JB, Alavi A, Hurtig HI, Zimmerman RA. MR signal abnormalities at 1.5 T in Alzheimer's dementia and normal aging. AJR Am J Roentgenol. 1987;149(2):351-6.

21. Scheltens P, Barkhof F, Leys D, Pruvo JP, Nauta JJ, Vermersch P, et al. A semiquantitative rating scale for the assessment of signal hyperintensities on magnetic resonance imaging. J Neurol Sci. 1993;114(1):7-12.

22. Beyer MK, Aarsland D, Greve OJ, Larsen JP. Visual rating of white matter hyperintensities in Parkinson's disease. Mov Disord. 2006;21(2):223-9.

23. Schiess MC, Zheng H, Soukup VM, Bonnen JG, Nauta HJ. Parkinson's disease subtypes: clinical classification and ventricular cerebrospinal fluid analysis. Parkinsonism Relat Disord. 2000;6(2):69-76.

24. Antonini A, Barone P, Marconi R, Morgante L, Zappulla S, Pontieri FE, et al. The progression of non-motor symptoms in Parkinson's disease and their contribution to motor disability and quality of life. J Neurol. 2012;259(12):2621-31.

25. Rupert G Jr. Simultaneous statistical inference. 2nd ed. New York: Springer Science \& Business Media; 2012.

26. Holford N, Nutt JG. Disease progression, drug action and Parkinson's disease: why time cannot be ignored. Eur J Clin Pharmacol. 2008;64(2):207-16.

27. Chahine LM, Siderowf A, Barnes J, Seedorff N, Caspell-Garcia C, Simuni $T$, et al. Predicting progression in Parkinson's disease using baseline and 1-year change measures. J Parkinsons Dis. 2019;9(4):665-79.

28. Raval V, Nguyen KP, Gerald A, Dewey RB Jr, Montillo A. Prediction of individual progression rate in Parkinson's disease using clinical measures and biomechanical measures of gait and postural stability. Proc IEEE Int Conf Acoust Speech Signal Process. 2020; 1319-1323.

29. Lewis MM, Harkins E, Lee EY, Stetter C, Snyder B, Corson T, et al. Clinical progression of Parkinson's Disease: insights from the NINDS common data elements. J Parkinsons Dis. 2020;10(3):1075-85.

30. Reinoso G, Allen JC Jr, Au WL, Seah SH, Tay KY, Tan LC. Clinical evolution of Parkinson's disease and prognostic factors affecting motor progression: 9-year follow-up study. Eur J Neurol. 2015;22(3):457-63.

31. Holden SK, Finseth T, Sillau SH, Berman BD. Progression of MDS-UPDRS scores over five years in De Novo Parkinson disease from the Parkinson's progression markers initiative cohort. Mov Disord Clin Pract. 2018;5(1):47-53

32. Santiago JA, Bottero V, Potashkin JA. Biological and clinical implications of comorbidities in Parkinson's disease. Front Aging Neurosci. 2017;9:394.

33. Saluja A, Parihar J, Garg D, Dhamija RK. The impact of COVID-19 pandemic on disease severity and quality of life in Parkinson's disease. Ann Indian Acad Neurol. 2021;24(2):217-26.

34. Shalash A, Roushdy T, Essam M, Fathy M, Dawood NL, Abushady EM, et al. Mental health, physical activity, and quality of life in Parkinson's disease during COVID-19 pandemic. Mov Disord. 2020;35(7):1097-9.

35. Douma EH, de Kloet ER. Stress-induced plasticity and functioning of ventral tegmental dopamine neurons. Neurosci Biobehav Rev. 2020:108:48-77.

36. Goldstein DS. Stress, allostatic load, catecholamines, and other neurotransmitters in neurodegenerative diseases. Cell Mol Neurobiol. 2012;32(5):661-6.

37. Chapuis S, Ouchchane L, Metz O, Gerbaud L, Durif F. Impact of the motor complications of Parkinson's disease on the quality of life. Mov Disord. 2005;20(2):224-30.

38. Abraham DS, Gruber-Baldini AL, Magder LS, McArdle PF, Tom SE, Barr E, et al. Sex differences in Parkinson's disease presentation and progression. Parkinsonism Relat Disord. 2019;69:48-54.

39. Iwaki H, Blauwendraat C, Leonard HL, Makarious MB, Kim JJ, Liu G, et al. Differences in the presentation and progression of Parkinson's disease by sex. Mov Disord. 2021;36(1):106-17.
40. Aleksovski D, Miljkovic D, Bravi D, Antonini A. Disease progression in Parkinson subtypes: the PPMI dataset. Neurol Sci. 2018;39(11):1971-6.

41. Ferguson LW, Rajput AH, Rajput A. Early-onset vs late-onset Parkinson's disease: a clinical-pathological study. Can J Neurol Sci. 2016;43(1):113-9.

42. Mollenhauer B, Zimmermann J, Sixel-Döring F, Focke NK, Wicke T, Ebentheuer J, et al. Baseline predictors for progression 4 years after Parkinson's disease diagnosis in the De Novo Parkinson Cohort (DeNoPa). Mov Disord. 2019;34(1):67-77.

43. Marras $C$, Rochon P, Lang AE. Predicting motor decline and disability in Parkinson disease: a systematic review. Arch Neurol. 2002;59(11):1724-8.

44. Paul KC, Chuang YH, Shih IF, Keener A, Bordelon Y, Bronstein JM, et al. The association between lifestyle factors and Parkinson's disease progression and mortality. Mov Disord. 2019;34(1):58-66.

45. Nag N, Jelinek GA. A narrative review of lifestyle factors associated with Parkinson's disease risk and progression. Neurodegener Dis. 2019;19(2):51-9.

46. Ayala A, Triviño-Juárez JM, Forjaz MJ, Rodríguez-Blázquez C, Rojo-Abuin JM, Martínez-Martín P. Parkinson's disease severity at 3 years can be predicted from non-motor symptoms at baseline. Front Neurol. 2017;8:551.

47. Chung SJ, Lee YH, Yoo HS, Oh JS, Kim JS, Ye BS, et al. White matter hyperintensities as a predictor of freezing of gait in Parkinson's disease. Parkinsonism Relat Disord. 2019:66:105-9.

48. Pozorski V, Oh JM, Okonkwo O, Krislov S, Barzgari A, Theisen F, et al. Cross-sectional and longitudinal associations between total and regional white matter hyperintensity volume and cognitive and motor function in Parkinson's disease. Neuroimage Clin. 2019;23: 101870.

49. Huo Y, Hong W, Huang J, Wang C, Ma J, Liu D, et al. White matter hyperintensities and the progression from mild parkinsonian signs to parkinsonism and Parkinson's disease. Neurobiol Aging. 2020;96:267-76.

50. Rektor I, Svátková A, Vojtišek L, Zikmundová I, Vaníček J, Király A, et al. White matter alterations in Parkinson's disease with normal cognition precede grey matter atrophy. PLoS ONE. 2018;13(1): e0187939.

51. Choe CU, Petersen E, Lezius S, Cheng B, Schulz R, Buhmann C, et al. Association of lipid levels with motor and cognitive function and decline in advanced Parkinson's disease in the Mark-PD study. Parkinsonism Relat Disord. 2021;85:5-10.

52. Sääksjärvi K, Knekt P, Männistö S, Lyytinen J, Heliövaara M. Prospective study on the components of metabolic syndrome and the incidence of Parkinson's disease. Parkinsonism Relat Disord. 2015;21(10):1148-55.

53. Fu $X$, Wang $Y$, He $X$, Li H, Liu H, Zhang X. A systematic review and metaanalysis of serum cholesterol and triglyceride levels in patients with Parkinson's disease. Lipids Health Dis. 2020;19(1):97.

54. Ascherio A, LeWitt PA, Xu K, Eberly S, Watts A, Matson WR, et al. Urate as a predictor of the rate of clinical decline in Parkinson disease. Arch Neurol. 2009;66(12):1460-8.

55. Sleeman I, Lawson RA, Yarnall AJ, Duncan GW, Johnston F, Khoo TK, et al. Urate and homocysteine: predicting motor and cognitive changes in newly diagnosed Parkinson's disease. J Parkinsons Dis. 2019;9(2):351-9.

56. Wu IC, Hsu CC, Chen CY, Chuang SC, Cheng CW, Hsieh WS, et al. Paradoxical relationship between glycated hemoglobin and longitudinal change in physical functioning in older adults: a prospective cohort study. J Gerontol A Biol Sci Med Sci. 2019;74(6):949-56.

\section{Publisher's Note}

Springer Nature remains neutral with regard to jurisdictional claims in published maps and institutional affiliations. 